\title{
To Do or Not to Do: The Neural Signature of Self-Control
}

\author{
Marcel Brass ${ }^{1,2}$ and Patrick Haggard ${ }^{3}$ \\ ${ }^{1}$ Max Planck Institute for Human Cognitive and Brain Sciences, 04103 Leipzig, Germany, ${ }^{2}$ Department of Experimental Psychology, Ghent University, 9000 \\ Ghent, Belgium, and ${ }^{3}$ Institute of Cognitive Neuroscience and Department of Psychology, University College London, London WC1N 3AR, United Kingdom
}

Voluntary action is fundamental to human existence. Recent research suggests that volition involves a specific network of brain activity, centered on the fronto-median cortex. An important but neglected aspect of intentional action involves the decision whether to act or not. This decision process is crucial in daily life because it allows us to form intentions without necessarily implementing them. In the present study, we investigate the neural correlates of intentionally inhibiting actions using functional magnetic resonance imaging. Our data show that a specific area of the fronto-median cortex is more strongly activated when people prepare manual actions but then intentionally cancel them, compared with when they prepare and then complete the same actions. Our results suggest that the human brain network for intentional action includes a control structure for self-initiated inhibition or withholding of intended actions. The mental control of action has an enduring scientific interest, linked to the philosophical concept of "free will." Our results identify a candidate brain area that reflects the crucial decision to do or not to do.

Key words: intentional processing; response inhibition; cognitive control; prefrontal cortex; fMRI; anterior insula

\section{Introduction}

The ability to form intentions and translate them into action is characteristically human. The human brain contains a network for intentional action, centered on the fronto-median cortex (Ball et al., 1999; Cunnington et al., 2002; Lau et al., 2004; Nachev et al., 2005). This network forms intentions about when and how to act (Libet et al., 1983; Haggard and Eimer, 1999) and also generates the conscious experience of intending (Fried et al., 1991; Lau et al., 2004). This network can partly be separated from brain areas that coordinate responses to external stimuli (Goldberg, 1985). Responding to external stimuli primarily involves a circuit including the parietal lobes and lateral premotor areas (Ammon and Gandevia, 1990). In contrast, when people make intentional actions that are endogenous rather than related to an immediate stimulus, a network of medial frontal areas is more strongly involved, including the presupplementary motor area (pre-SMA) and the cingulate motor area (CMA) (Ball et al., 1999; Cunnington et al., 2002; Lau et al., 2004; Nachev et al., 2005).

Human action generally involves selection. In the case of intentional action, the selection is internally generated, rather than specified by external stimuli. Previous studies focused on selecting when to perform a specific action (Jahanshahi et al., 1995; Jenkins et al., 2000; Nachev et al., 2005) or on how to achieve a desired action goal by selecting between alternative possible movements (Dirnberger et al., 1998; Lau et al., 2004; Waszak et al., 2005). However, few studies have considered the more fundamental process of selecting whether to make an action or not.

Received March 1, 2007; revised May 30, 2007; accepted June 25, 2007.

This work was supported by the German Research Foundation. We thank Bettina Johst for programming the experiment.

Correspondence should be addressed to Dr. Marcel Brass, Department of Experimental Psychology, Ghent University, Henri Dunantlaan 2,9000 Gent, Belgium. E-mail: marcel.brass@ugent.be.

D01:10.1523/JNEUROSCI.0924-07.2007

Copyright $\odot 2007$ Society for Neuroscience $\quad$ 0270-6474/07/279141-05\$15.00/0
Nevertheless, this decision whether to act often has critical consequences. For example, there is a clear distinction between intending to hit someone and actually hitting them. Many human societies acknowledge this distinction by requiring both physical action (the actus reus of Roman Law) and conscious intention (mens rea) to attribute legal and moral responsibility. Humans therefore have a widely acknowledged ability to make or withhold actions, on which society and culture rely but the neural basis of which remains poorly understood.

The frontal lobes have previously been identified with inhibition of responses to external stimuli (Konishi et al., 1998; Rubia et al., 2001; Aron et al., 2003). However, it remains unclear whether the endogenous decision to execute or inhibit an intended action involves an analogous system for endogenous inhibition of intentional action. In the present experiment, we investigated the brain processes associated with endogenous inhibition using an established method for reporting the conscious experience of intentional action in combination with event-related functional magnetic resonance imaging ( $\mathrm{fMRI}$ ).

\section{Materials and Methods}

Participants. Nineteen participants were recruited in a behavioral preexperiment. We selected 15 participants on the basis of this preexperiment. Selection was not based on behavioral data but on an informal interview in which we asked participants how they performed the task and followed the instructions. Four subjects reported that they were not able to follow the instructions and were excluded. The remaining 15 subjects (seven males and eight females; mean age, 26.06 years) had normal or corrected-to-normal vision. None of the subjects had a history of neurological, major medical, or psychiatric disorders. All were right handed, as assessed by the Edinburgh Inventory. One additional subject was excluded because of excessive head movement during scanning.

Design. In the present experiment (Fig. 1) participants were instructed to freely decide when to execute a simple key press while observing a rotating clock hand (Libet et al., 1983). Furthermore, they were told to cancel the intended response at the last possible moment in some trials 
that they freely selected. After each trial, they had to determine the clock position when they formed the intention to act. Our interest focused on the comparison between trials in which subjects intentionally inhibited the action and trials in which they intentionally executed it.

The fMRI experiment consisted of six experimental blocks with 20 trials each. In four blocks, participants had to press a key (action condition) or inhibit the key press (inhibition condition). In the other two blocks, participants had to respond to a tone (tone condition).

Each trial began with a clock face appearing on the screen. Participants initiated the trial by pressing a key with their left index finger. After a random delay of 500-2000 ms, the clock hand appeared at a random position and began to rotate once (period, $3000 \mathrm{~ms}$ ). The clock hand always made at least one full turn. In the action and inhibition trials, participants had two additional rotation cycles to initiate the key press (action trials) or intend the key press and afterward inhibit it (inhibition). After a key press in the action condition, the clock hand continued to rotate for another 1500-3000 ms and then disappeared. In inhibition trials, the hand simply disappeared after three rotations. Participants reported the time point when they decided to act by using a trackball to reposition a cursor at the relevant position on the clock face. They operated with their right hand. Participants had a $10 \mathrm{~s}$ time period to operate the trackball. In tone blocks, participants started the trial as in action blocks. However, in this condition, a tone was presented during the two rotations after the first rotation. The timing of the tone was yoked to the key-press action in action trials. Participants did not make voluntary key presses and had to indicate the onset of the tone with the trackball. Other details were as for action trials. After each trial, a random interval between 1000 and $3000 \mathrm{~ms}$ occurred before the next trial started.

MRI scanning procedure. The experiment was performed on a 3T scanner (Medspec 30/100; Bruker, Ettlingen, Germany). Twenty axial slices (19.2 cm field of view, $64 \times 64$ matrix, $4 \mathrm{~mm}$ thickness, $1 \mathrm{~mm}$ spacing), parallel to the anterior commissure-posterior commissure plane and covering the entire brain, were acquired using a single-shot, gradientrecalled echo planar imaging (EPI) sequence (repetition time, $2000 \mathrm{~ms}$; echo time, $30 \mathrm{~ms}, 90^{\circ}$ flip angle). Before the functional runs, 20 corresponding anatomical modified driven equilibrium Fourier transformation (MDEFT) slices and 20 EPI-T1 slices were acquired. Stimuli were displayed by a liquid crystal display projector on a backprojection screen mounted in the bore of the magnet behind the participants' head.

fMRI analysis. Analysis of fMRI data was performed using the LIPSIA software package. Data were filtered using a spatial Gaussian filter with $\sigma=1$. A temporal high-pass filter with a cutoff frequency of $1 / 80 \mathrm{~Hz}$ was used for baseline correction. In addition, a global scaling was performed. All functional data sets were individually registered into threedimensional space using the participants' individual high-resolution anatomical images. The two-dimensional anatomical MDEFT slices, geometrically aligned with the functional slices, were used to compute a transformation matrix containing rotational and translational parameters that register the anatomical slices with the three-dimensional reference $\mathrm{T} 1$ data set. These transformation matrices were normalized to the standard Talairach brain size by linear scaling and finally applied to the individual functional data. The statistical evaluation was performed using the general linear model for serially autocorrelated observations. The design matrix for event-related analysis was created using a model of the hemodynamic response with a variable delay. The trigger for the eventrelated analysis was the subject's judgment of the time at which they experienced the intention to act, in the case of action and inhibition trials, or the judgment of tone onset in the case of tone trials. In inhibition trials, the temporal judgment was ambiguous because it could be related to the first or second rotation of the clock hand in which the subject was instructed to perform actions. Therefore, we put the trigger in the middle of both possible time points. The model equation was convolved with a Gaussian kernel with a dispersion of $4 \mathrm{~s}$ full-width at half-maximum. Contrast maps were generated for each participant. A one-sample $t$ test of contrast maps across participants (random-effects model) was computed to ascertain whether observed differences between conditions were significantly different. Subsequently, $t$ values were transformed into $z$ scores. We only report cortical regions with a $z<0.001$ and a volume size lager than $225 \mathrm{~mm}^{3}$ corresponding to five measured adjacent voxels $(3 \times$ $3 \times 5 \mathrm{~mm}$ ).

\section{Results}

Subjects reported the subjective experience of deciding to initiate action a mean of $-141 \mathrm{~ms}$ before the key press on action trials (Libet et al., 1983). The mean proportion of inhibition trials was $45.5 \%$, but there were large interindividual differences, with the proportion of inhibition trials ranging from 28 to $62 \%$.

To determine the brain areas that were involved in inhibition of intentional action, we contrasted inhibition trials with action trials. This contrast yielded activation in the dorsal frontomedian cortex (dFMC; Brodmann's area 9) (Fig. 2A), the left and right anterior ventral insula (Fig. $2 B$ ), and the right superior temporal sulcus (STS) (Table 1).

The reverse contrast between action trials and inhibition trials yielded activation in a number of motor-related areas such as the primary sensorimotor cortex and the cerebellum but no activation in medial or lateral prefrontal cortex. The absence of any significant difference in areas known to be involved in preparation of voluntary action, such as pre-SMA and SMA (Cunnington et al., 2003), suggests that subjects prepared intentional actions equally both in the action trials and in the inhibition trials. This is consistent with previous reports of comparable readiness potentials in action and in inhibition trials. Our conclusion was also supported by separately comparing both action and inhibition trials with control "tone" trials in which subjects never prepared or made actions. Both contrasts yielded a significant $(p<0.001)$ activation focus in the SMA/pre-SMA. The peak of the preparation-related activation was in a very similar location for the action trials $(x=-1, y=-5, z=59)$ and for the inhibition trials $(x=4, y=0, z=62)$. Parameter estimates for the pre-SMA $(x=-1, y=-5, z=59)$ show no significant difference in activation strength for the action and inhibition condition $\left(t_{(13)}\right.$ $=0.76 ; p=0.45$ ) (Fig. 3A). Moreover, this preparation-related 


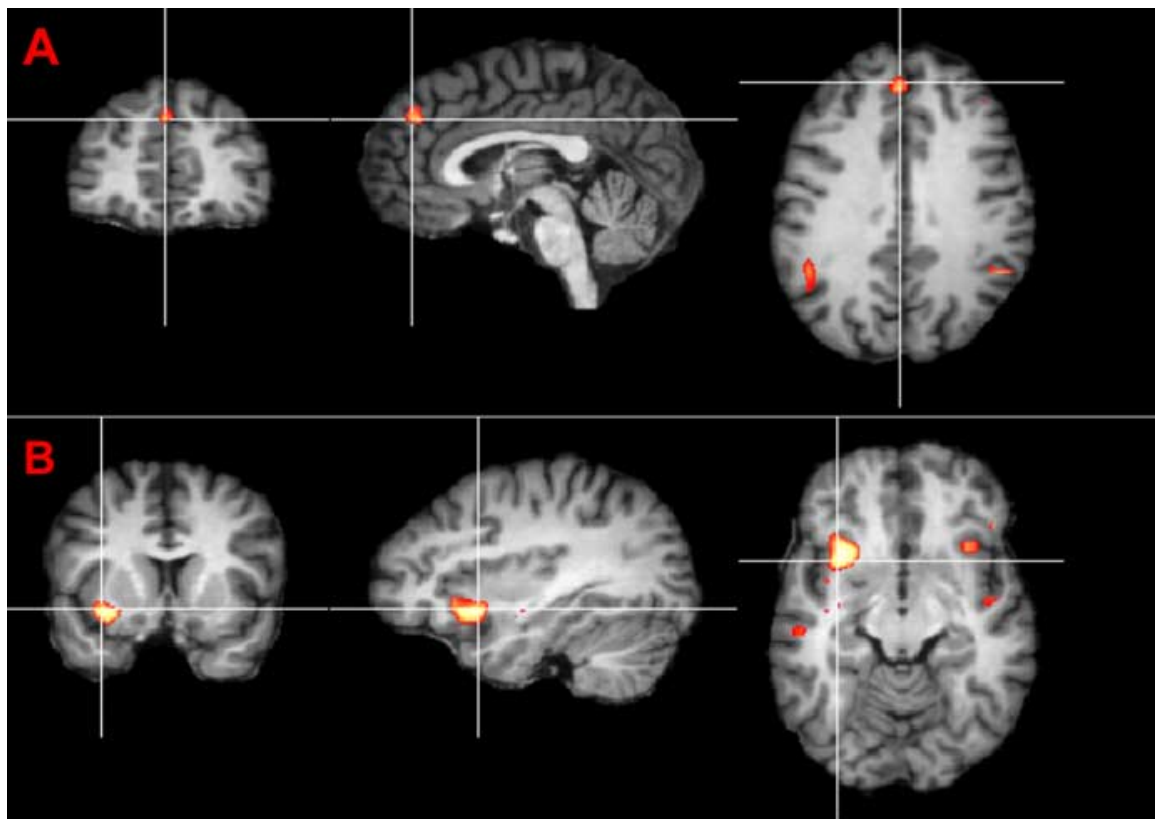

Figure 2. A, Activation in the dFMC for the contrast of inhibition versus action trials. The $z$-map is thresholded at $z>3.09$ $(p<0.001)$. $\boldsymbol{B}$, Activation in the left anterior ventral insula for the contrast of inhibition versus action trials.

Table 1. Anatomical location of brain activation for inhibition versus action

\begin{tabular}{llll}
\hline Anatomical location & Maximum $z$ value & Laterality & Talairach coordinates $(x, y, z)$ \\
\hline dFMC & 3.75 & Left & $-2,41,32$ \\
Insula & 4.71 & Left & $-31,8,-6$ \\
Insula & 3.61 & Right & $32,17,-9$ \\
STS & 3.44 & Left & $-53,-25,-3$ \\
\hline
\end{tabular}
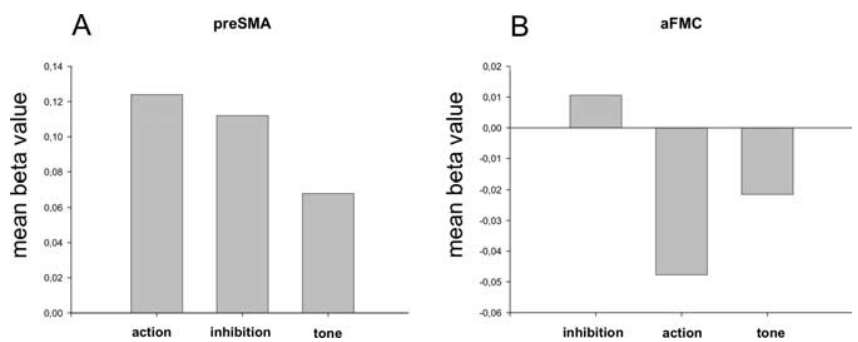

Figure 3. $A$, Parameter estimates for the blood oxygenation level-dependent (BOLD) signal in the pre-SMA for the three experimental conditions (inhibition, action, tone). $\boldsymbol{B}$, Parameter estimates for the BOLD signal in the anterior fronto-median cortex (aFMC) for the three experimental conditions (inhibition, action, tone).

activation was spatially quite distinct from the dFMC activation found when contrasting inhibition trials with action trials $(x=$ $-1, y=42, z=32$ ).

We hypothesized that the level of neural activity in any putative area for endogenous inhibition should increase the probability of inhibiting action. We therefore correlated each subject's percentage of inhibition trials with their inhibition-related activation, defined as the difference between $\mathrm{dFMC}$ activations for inhibition and action trials. This showed a positive correlation ( $r=0.47 ; p<0.05$, one-tailed): participants with stronger inhibition-related dFMC activation inhibited actions more frequently.

Finally, we were interested in the relationship between inhibition-related dFMC activation and activation of primary motor cortex. If the dFMC is critical for the endogeneous inhibi- tion of action, then its activation should be negatively related to activation of motor execution areas, such as primary motor cortex. Therefore, we expected a negative correlation for the inhibition-related dFMC activation and the activation in primary motor cortex in inhibition trials. Furthermore, this correlation should be modulated by the success of the putative inhibition process: strong inhibition should both increase the number of inhibition trials and strengthen the relationship between dFMC and primary motor activation. Activation in the dFMC and the primary motor cortex were significantly negatively correlated across subjects when we controlled for each subject's number of inhibition trials (partial $r=-0.65 ; p<$ $0.01)$.

\section{Discussion}

Our data suggest that inhibition of intentional actions involves a fronto-median cortical area. This area is distinct from the areas that generate intentional actions, attend to intentions, or select between alternatives. A number of studies have found the SMA/pre-SMA and the CMA to be involved in intentional action (Ball et al., 1999; Cunnington et al., 2002; Lau et al., 2004; Nachev et al., 2005). However, the inhibition-related activation in the present study is too dorsal to be considered as part of the CMA and clearly too anterior to be related to the SMA/pre-SMA complex, as indicated by probabilistic cytoarchitectonic maps (Eickhoff et al., 2005).

\section{The role of the dFMC in intentional inhibition of action}

Cognitive models of inhibition have focused on inhibition of prepotent responses to external stimuli (Logan et al., 1984; Cohen et al., 1990). An important distinction is made between "lateral" competitive interaction between alternative representations at a single level (Rumelhart and McClelland, 1986) and inhibitory top-down control signals from hierarchically higher brain areas (Norman and Shallice, 1986). The first idea would be consistent with a general decision process being involved. If the AFMC decides between action and inhibition by a competitive interaction process, then representations corresponding to the possibilities of action and to non-action should initially both be active, leading to activation in both action trials and inhibition trials. Our finding of minimal dFMC activation in action trials (Fig. $3 B$ ) argues against a view of endogenous inhibition based on competitive interaction between alternatives and thus is also not consistent with the idea of the dFMC being involved in a general decision process. In contrast, our result is consistent with a specific top-down control signal gating the neural pathways linking intention to action. This view is supported by the negative correlation between dFMC activation and primary motor cortex activation.

This point is also supported by the anatomical finding that activation related to inhibition was located more anteriorly than the activation related to intentional action. The prefrontal cortex shows an anterior-posterior functional gradient (Dreher et al., 2002; Forstmann et al., 2005). More anterior regions are related to higher-order intentionality, and more posterior regions are more closely related to generating the physical action itself. We 
therefore suggest that inhibition of intentional action involves processes that are located higher in the hierarchy of control than the development of the immediate action plan itself. Moreover, the areas involved in inhibition of intentional action have been identified by meta-analyses as playing a wider role in evaluation and appraisal of material related to the self, including but not restricted to motor acts (Northoff and Bermpohl, 2004).

We also found a positive correlation across individuals between inhibition-related dFMC activation and the frequency of inhibited actions. In the psychology of individual differences, disinhibited and impulsive behavior is a crucial marker for specific personality traits that have been related to antisocial and criminal behavior (Swann et al., 2002). However, the brain mechanisms involved in such behavior are still poorly understood. Our findings suggest specific brain regions may be involved. Furthermore, inhibition of intentional actions may be closer to the unconstrained situations of everyday action than classical response inhibition paradigms generally used to investigate such behavior (Horn et al., 2003).

\section{The anterior insula and the feeling of "let down"}

Although we expected an activation in the fronto-median cortex for the inhibition condition, the activations of the ventral insula and the STS were not predicted. Anterior insula activation has been reported in response inhibition studies (Garavan et al., 1999; Wager et al., 2005), but usually more dorsally than here. Furthermore, an activation very close to the present activation located in the mid-ventrolateral prefrontal cortex has been related to negative feedback in a Wisconsin Card Sorting task (Monchi et al., 2001). Meta-analytic data suggest that the ventral insula is involved in emotional rather than cognitive processes (Wager and Feldman Barrett, 2004).

This area may evaluate afferent homeostatic information from the body, leading to "subjective feeling states arising from representations of bodily responses" (Damasio, 1999; Critchley et al., 2004). Furthermore, the anterior insula has been found to be related to the feeling of frustration (Abler et al., 2005). Canceling an ongoing motor intention may result in an unresolved tension or feeling of let down. A recent study using the stop-signal paradigm is consistent with this interpretation. Ramautar et al. (2006) found stronger insula activation on unsuccessful stopsignal trials for blocks with rare stop signals compared with more frequent stop signals. They attributed this difference to the stronger feeling of failure in the rare condition. This description captures nicely the report of our debriefing in which most participants reported inhibition trials to be extremely frustrating. We suggest that insula activation represents the affective-somatic consequences of failing to implement a strong intention.

\section{Intentional inhibition as a crucial component of intentional action}

The inhibition of intentional action has been primarily neglected both in the neuroscientific literature on response inhibition and in that on intentional action. Our findings suggest that inhibition of intentional action involves cortical areas different from, and upstream from, the intentional generation and execution of action. In addition, this process of "last-minute" inhibition is compatible with a conscious experience of intending to act. Some parts of the inhibition process may occur after the intention to perform an action has become conscious. For example, Libet (Libet et al., 1983; Libet, 2004) hypothesized a "veto" allowing the conscious mind to intervene to withhold unconsciously initiated action plans. Because he could not find any identifiable neural correlate of the veto process, he suggested it could involve mindbrain causation: a "free won't" analogous to "free will." Our data identify a clear neural basis for inhibiting intentions and thus identify the neural correlate of the veto process. The hypothesis of a special, non-neural veto process could therefore become unnecessary. Because the initiation of action is unconscious (Libet et al., 1983), then the initiation of inhibition may also be unconscious. In addition, the poor temporal resolution of fMRI means we cannot yet judge whether the inhibitory process could operate in the seemingly brief interval between the time of conscious intention and the point of no return for motor output (Libet et al., 1983; Walton et al., 2004). Our results provide the first clear neuroscientific basis for the widely held view that people can refrain from doing something even if they genuinely wish to do it (Baumeister and Exline, 1999). We speculate that the dFMC may be involved in those aspects of behavior and personality that reflect "self-control" (Northoff and Bermpohl, 2004). Identifying specific brain processes and regions that are central to individuals' personalities, and are also of social and legal importance, may have profound neuroethical implications.

\section{References}

Abler B, Walter H, Erk S (2005) Neural correlates of frustration. NeuroReport 16:669-672.

Ammon K, Gandevia SC (1990) Transcranial magnetic stimulation can influence the selection of motor programmes. J Neurol Neurosurg Psychiatry 53:705-707.

Aron AR, Fletcher PC, Bullmore ET, Sahakian BJ, Robbins TW (2003) Stopsignal inhibition disrupted by damage to right inferior frontal gyrus in humans. Nat Neurosci 6:115-116.

Ball T, Schreiber A, Feige B, Wagner M, Lucking CH, Kristeva-Feige R (1999) The role of higher-order motor areas in voluntary movement as revealed by high-resolution EEG and fMRI. NeuroImage 10:682-694.

Baumeister RF, Exline JJ (1999) Virtue, personality, and social relations: self-control as the moral muscle. J Pers 67:1165-1194.

Cohen JD, Dunbar K, McClelland JL (1990) On the control of automatic processes: a parallel distributed processing account of the Stroop effect. Psychol Rev 97:332-361.

Critchley HD, Wiens S, Rotshtein P, Ohman A, Dolan RJ (2004) Neural systems supporting interoceptive awareness. Nat Neurosci 7:189-195.

Cunnington R, Windischberger C, Deecke L, Moser E (2002) The preparation and execution of self-initiated and externally-triggered movement: a study of event-related fMRI. NeuroImage 15:373-385.

Cunnington R, Windischberger C, Deecke L, Moser E (2003) The preparation and readiness for voluntary movement: a high-field event-related fMRI study of the Bereitschafts-BOLD response. NeuroImage 20:404-412.

Damasio A (1999) The feeling of what happens: body, emotion and the making of consciousness. London: Heinemann.

Dirnberger G, Fickel U, Lindinger G, Lang W, Jahanshahi M (1998) The mode of movement selection. Movement-related cortical potentials prior to freely selected and repetitive movements. Exp Brain Res 120:263-272.

Dreher JC, Koechlin E, Ali SO, Grafman J (2002) The roles of timing and task order during task switching. NeuroImage 17:95-109.

Eickhoff SB, Stephan KE, Mohlberg H, Grefkes C, Fink GR, Amunts K, Zilles K (2005) A new SPM toolbox for combining probabilistic cytoarchitectonic maps and functional imaging data. NeuroImage 25:1325-1335.

Forstmann BU, Brass M, Koch I, von Cramon DY (2005) Internally generated and directly cued task sets: an investigation with fMRI. Neuropsychologia 43:943-952.

Fried I, Katz A, McCarthy G, Sass KJ, Williamson P, Spencer SS, Spencer DD (1991) Functional organization of human supplementary motor cortex studied by electrical stimulation. J Neurosci 11:3656-3666.

Garavan H, Ross TJ, Stein EA (1999) Right hemispheric dominance of inhibitory control: an event-related functional MRI study. Proc Natl Acad Sci USA 96:8301-8306.

Goldberg G (1985) Supplementary motor area structure and function: review and hypothesis. Behav Brain Sci 8:567-616.

Haggard P, Eimer M (1999) On the relation between brain potentials and the awareness of voluntary movements. Exp Brain Res 126:128-133. 
Horn NR, Dolan M, Elliott R, Deakin JF, Woodruff PW (2003) Response inhibition and impulsivity: an fMRI study. Neuropsychologia 41:1959-1966.

Jahanshahi M, Jenkins IH, Brown RG, Marsden CD, Passingham RE, Brooks DJ (1995) Self-initiated versus externally triggered movements. I. An investigation using measurement of regional cerebral blood flow with PET and movement-related potentials in normal and Parkinson's disease subjects. Brain 118:913-933.

Jenkins IH, Jahanshahi M, Jueptner M, Passingham RE, Brooks DJ (2000) Self-initiated versus externally triggered movements. II. The effect of movement predictability on regional cerebral blood flow. Brain 123:1216-1228.

Konishi S, Nakajima K, Uchida I, Sekihara K, Miyashita Y (1998) No-go dominant brain activity in human inferior prefrontal cortex revealed by functional magnetic resonance imaging. Eur J Neurosci 10:1209-1213.

Lau HC, Rogers RD, Haggard P, Passingham RE (2004) Attention to intention. Science 303:1208-1210.

Libet B, ed (2004) Mind time. The temporal factor in consciousness. Cambridge, MA: Harvard UP.

Libet B, Gleason CA, Wright EW, Pearl DK (1983) Time of conscious intention to act in relation to onset of cerebral activity (readiness-potential). The unconscious initiation of a freely voluntary act. Brain 106:623-642.

Logan GD, Cowan WB, Davis KA (1984) On the ability to inhibit simple and choice reaction time responses: a model and a method. J Exp Psychol Hum Percept Perform 10:276-291.

Monchi O, Petrides M, Petre V, Worsley K, Dagher A (2001) Wisconsin Card Sorting revisited: distinct neural circuits participating in different stages of the task identified by event-related functional magnetic resonance imaging. J Neurosci 21:7733-7741.

Nachev P, Rees G, Parton A, Kennard C, Husain M (2005) Volition and conflict in human medial frontal cortex. Curr Biol 15:122-128.
Norman D, Shallice T (1986) Attention to action: willed and automatic control of behavior. In: Consciousness and self regulation: advances in research and theory (Davidson R, Schwartz G, Shapiro D, eds), pp 1-18. New York: Plenum.

Northoff G, Bermpohl F (2004) Cortical midline structures and the self. Trends Cogn Sci 8:102-107.

Ramautar JR, Slagter HA, Kok A, Ridderinkhof KR (2006) Probability effects in the stop-signal paradigm: the insula and the significance of failed inhibition. Brain Res 1105:143-154.

Rubia K, Russell T, Overmeyer S, Brammer MJ, Bullmore ET, Sharma T, Simmons A, Williams SC, Giampietro V, Andrew CM, Taylor E (2001) Mapping motor inhibition: conjunctive brain activations across different versions of go/no-go and stop tasks. NeuroImage 13:250-261.

Rumelhart D, McClelland J, eds (1986) Parallel distributed processing. Cambridge, MA: MIT.

Swann AC, Bjork JM, Moeller FG, Dougherty DM (2002) Two models of impulsivity: relationship to personality traits and psychopathology. Biol Psychiatry 51:988-994.

Wager TD, Feldman Barrett L (2004) From affect to control: functional specialization of the insula in motivation and regulation. Retrieved $\mathrm{Au}$ gust 9, 2006 from http://www.columbia.edu/cu/psychology/tor/Papers/ Wager_Feldman_Barrett_2004_Insula_meta-analysis.pdf.

Wager TD, Sylvester CY, Lacey SC, Nee DE, Franklin M, Jonides J (2005) Common and unique components of response inhibition revealed by fMRI. NeuroImage 27:323-340.

Walton ME, Devlin JT, Rushworth MF (2004) Interactions between decision making and performance monitoring within prefrontal cortex. Nat Neurosci 7:1259-1265.

Waszak F, Wascher E, Keller P, Koch I, Aschersleben G, Rosenbaum DA, Prinz W (2005) Intention-based and stimulus-based mechanisms in action selection. Exp Brain Res 162:346-356. 